\title{
Permutation Groups with a Cyclic Regular Subgroup and Arc Transitive Circulants
}

CAI HENG LI

li@maths.uwa.edu.au

Department of Mathematics, Yunnan University, Kunming 650031, People's Republic of China;

School of Mathematics and Statistics, The University of Western Australia, Crawley, 6009 WA, Australia

Received January 29, 2003; Revised January 21, 2004; Accepted January 21, 2004

Abstract. A description is given of finite permutation groups containing a cyclic regular subgroup. It is then applied to derive a classification of arc transitive circulants, completing the work dating from 1970's. It is shown that a connected arc transitive circulant $\Gamma$ of order $n$ is one of the following: a complete graph $\mathrm{K}_{n}$, a lexicographic product $\Sigma\left[\overline{\mathrm{K}}_{b}\right]$, a deleted lexicographic product $\Sigma\left[\overline{\mathrm{K}}_{b}\right]-b \Sigma$, where $\Sigma$ is a smaller arc transitive circulant, or $\Gamma$ is a normal circulant, that is, Aut $\Gamma$ has a normal cyclic regular subgroup. The description of this class of permutation groups is also used to describe the class of rotary Cayley maps in subsequent work.

Keywords: cyclic regular subgroup, arc transitive circulant

1991 MR Subject Classification: 20B15, 20B30, 05C25

\section{Introduction}

A finite permutation group which contains a cyclic regular subgroup is called a $c$-group, for convenience. Characterizing $c$-groups is an old topic in permutation group theory, initiated by Burnside (1900), and studied by Schur et al., see for example [17, Chapter 4], [5], [7, Theorem 1.49] and [9]. The classical result of Schur tells us that a primitive $c$-group is 2-transitive or has prime degree. Thus, based on the finite simple group classification, primitive $c$-groups are essentially known in 1980's, see [5, 7, 9]. A precise list of primitive $c$-groups was recently given in $[8,13]$, independently.

Proposition 1.1 (see [13, Corollary 1.2]) A primitive permutation group $X$ of degree $n$ contains a cyclic regular subgroup $G$ if and only if one of the following holds:

(i) $\mathbb{Z}_{p} \cong G \leq X \leq \operatorname{AGL}(1, p)$, where $p$ is a prime;

(ii) $X=\mathrm{A}_{n}$ with $n$ odd, or $\mathrm{S}_{n}$, where $n \geq 4$;

(iii) $\mathrm{PGL}(d, q) \leq X \leq \mathrm{P} \Gamma \mathrm{L}(d, q)$, and $G$ is a Singer subgroup of $X$, and $n=\frac{q^{d}-1}{q-1}$;

(iv) $X=\mathrm{P} \Gamma \mathrm{L}(2,8)$, and $G=\langle s \sigma\rangle \cong \mathbb{Z}_{9}$, where $\langle s\rangle$ is a Singer subgroup of $X$ and $\sigma \in X \backslash \mathrm{PSL}(2,8)$ such that $o(\sigma)=3$;

(v) $(X, n)=(\operatorname{PSL}(2,11), 11),\left(\mathrm{M}_{11}, 11\right)$, or $\left(\mathrm{M}_{23}, 23\right)$.

The main purpose of this paper is to give a description of general $c$-groups. To state the description, we need more notation and definitions. 
For a transitive permutation group $X$ on $\Omega$, an orbit $\Delta$ of $X$ on $\Omega \times \Omega$ is called an orbital, and the digraph with vertex set $\Omega$ and arc set $\Delta$ is called an orbital graph of $X$. (An arc of a digraph is an ordered pair of adjacent vertices.) An arc-disjoint union of orbital graphs is called a generalised orbital graph of $X$.

As usual, denote by $\mathrm{K}_{n}$ the complete graph of order $n$. By $\overline{\mathrm{K}}_{n}$ we mean the complement of $\mathrm{K}_{n}$, that is, the graph with the same vertex as $\mathrm{K}_{n}$ and contains no edges. For a digraph $\Gamma$ with vertex set $V$ and a digraph $\Sigma$ with vertex set $W$, the lexicographic product $\Gamma[\Sigma]$ of a digraph $\Sigma$ by a digraph $\Gamma$ is the digraph with vertex set $V \times W$ such that $\left(v_{1}, w_{1}\right)$ is adjacent to $\left(v_{2}, w_{2}\right)$ if and only if either $v_{1}$ is adjacent to $v_{2}$ in $\Gamma$, or $v_{1}=v_{2}$ and $w_{1}$ is adjacent to $w_{2}$ in $\Sigma$.

Let $\Gamma$ be a digraph with vertex set $\Omega$ such that $X \leq$ Aut $\Gamma$ is transitive on $\Omega$. For a normal subgroup $N \triangleleft X$ which is intransitive on $\Omega$, denote by $\Omega_{N}$ the set of $N$-orbits in $\Omega$. The normal quotient $\Gamma_{N}$ of the digraph $\Gamma$ induced by $N$ is the digraph with vertex set $\Omega_{N}$ such that for any $B, C \in \Omega_{N}, B$ is adjacent to $C$ if and only if there exist $u \in B$ and $v \in C$ such that $u$ is adjacent to $v$ in $\Gamma$.

A $c$-group $X$ is called a normal c-group if it contains a normal regular cyclic group. Then by Proposition 1.1, a primitive $c$-group is either 2-transitive, or normal. In particular, all insoluble primitive $c$-groups are 2-transitive.

A description of $c$-groups is stated in the following theorem, which shows that either the structure of $X$ is known, or the structure of some orbital graphs of $X$ is known.

Theorem 1.2 Let $X$ be a permutation group on a set $\Omega$ of degree $n$. Assume that $X$ contains a cyclic regular subgroup $G$. Then one of the following holds:

(1) $X$ has a normal subgroup $Y=Y_{1} \times Y_{2} \times \cdots \times Y_{r}$ with $r \geq 1$ such that $G \leq Y$, and further,

(i) each $Y_{i}$ is a 2-transitive c-group or a normal c-group of degree $n_{i}$,

(ii) $n=n_{1} n_{2} \ldots n_{r}$, and the $n_{i}$ are pairwise coprime.

(2) X has a normal subgroup $N$ such that each connected generalised orbital graph of $X$ contains a subgraph $\Gamma$ which is an orbital graph of $X$ and is of the form $\Gamma=\Gamma_{N}\left[\overline{\mathrm{K}}_{b}\right]$, where $b=|N \cap G|$.

A digraph $\Gamma$ is called a Cayley graph if there exist a group $G$ and a subset $S \subset G$ such that the vertex set of $\Gamma$ may be identified with $G$ and $u$ is adjacent to $v$ if and only if $v u^{-1} \in S$. This digraph $\Gamma$ is denoted by $\operatorname{Cay}(G, S)$. A Cayley graph $\Gamma=\operatorname{Cay}(G, S)$ has an automorphism group

$$
\hat{G}=\{\hat{g}: x \mapsto x g \text { for all } x \in G \mid g \in G\},
$$

consisting all right multiplications of elements of $G$. Thus the subgroup $\hat{G} \leq$ Aut $\Gamma$ acts regularly on the vertex set of $\Gamma$. In particular, $\Gamma$ is vertex transitive. Let $\operatorname{Aut}(G, S)=\{\sigma \in$ $\left.\operatorname{Aut}(G) \mid S^{\sigma}=S\right\}$. Then each element in $\operatorname{Aut}(G, S)$ induces an automorphism of the Cayley graph $\Gamma=\operatorname{Cay}(G, S)$. Moreover, it is easily shown that $\mathbf{N}_{\mathrm{Aut} \Gamma}(\hat{G})=\hat{G}: \operatorname{Aut}(G, S)$, see for example [6]. A Cayley graph $\Gamma=\operatorname{Cay}(G, S)$ is called normal if $\hat{G}$ is normal in Aut $\Gamma$, that is, Aut $\Gamma=\hat{G}: \operatorname{Aut}(G, S)$. 
Let $G$ be a cyclic group, and let $\Gamma=\operatorname{Cay}(G, S)$. Then this Cayley graph is called a circulant. Since Aut $\Gamma$ contains the cyclic regular subgroup $\hat{G}$, Aut $\Gamma$ is a $c$-group. If $\Gamma$ is a normal Cayley graph, that is, $\hat{G}$ is normal in Aut $\Gamma$, then $\Gamma$ is called a normal circulant. The description of $c$-groups given in Theorem 1.2 can be used to study circulants. The second result of this paper is to present a classification of arc transitive circulants. (Recall that a graph $\Gamma$ is arc transitive if Aut $\Gamma$ is transitive on the arcs of $\Gamma$.)

The problem of classifying arc transitive circulants has been investigated for a long time, dating from 1970's, and it has been solved for several special cases: A classification of 2-arc transitive circulants was obtained in [1] for the undirected case and in [16] for the directed case; while a classification of arc transitive circulants of special orders was given by a collection of articles: prime order in [2,3], square-free order in [15], and odd prime-power order in [19].

For a positive integer $b$ and a digraph $\Gamma$, denote by $b \Gamma$ the digraph consisting of $b$ vertexdisjoint copies of $\Gamma$; the digraph $\Gamma\left[\overline{\mathrm{K}}_{b}\right]-b \Gamma$ is called a deleted lexicographic product, which is the digraph whose vertex set is the vertex set of $\Gamma\left[\bar{K}_{b}\right]$ and arc set equals the arc set of $\Gamma\left[\overline{\mathrm{K}}_{b}\right]$ minus the arc set of $b \Gamma$.

Theorem 1.3 Let $\Gamma$ be a connected arc transitive circulant of order $n$ which is not a complete graph. Then either

(1) $\Gamma$ is a normal circulant, or

(2) there exists an arc transitive circulant $\Sigma$ of order $m$ such that $n=m b$ with $b, m>1$ and

$$
\Gamma=\left\{\begin{array}{l}
\Sigma\left[\overline{\mathrm{K}}_{b}\right], \quad \text { or } \\
\Sigma\left[\overline{\mathrm{K}}_{b}\right]-b \Sigma \quad \text { with }(b, m)=1
\end{array}\right.
$$

\section{Remarks}

(a) After the previous version of this paper had been submitted for publication, the author was aware of that the classification of arc transitive circulants given in Theorems 1.3 was also obtained by I. Kovács [10], independently. Also the referees of this paper pointed out this to the author.

(b) It is easily shown that a digraph is a circulant if and only if its automorphism group is a $c$-group. An arc transitive circulant is therefore exactly an orbital graph of a $c$ group. Thus the proof of Theorem 1.3 easily follows from Theorem 1.2, and will not be presented in this paper. A proof for this classification may be found in [10].

(c) If an arc transitive circulant $\Gamma$ is of the form $\Sigma\left[\overline{\mathrm{K}}_{b}\right]$ or $\Sigma\left[\overline{\mathrm{K}}_{b}\right]-b \Sigma$, namely $\Gamma$ satisfies part (2) of Theorem 1.3, then $\Gamma$ can be easily reconstructed from a smaller arc transitive circulant $\Sigma$. As the automorphism group of a cyclic group is abelian, normal circulants may be easily and explicitly constructed. Arc transitive circulants are therefore wellcharacterized by Theorem 1.3.

(d) The circulants concerned in Theorem 1.3 are directed graphs. It is easily shown that an undirected edge transitive circulant $\Gamma$ is arc transitive, and Theorem 1.3 may be easily changed into a version for the undirected case. 


\section{Proof of Theorem 1.2}

This section is devoted to proving Theorem 1.2. The proof is based on a result of Evdokimov and Ponomarenko [4] regarding the 2-closures of $c$-groups. We here first introduce some simple properties for the 2-closures of transitive permutation groups.

Let $G$ be a transitive permutation group on $\Omega$. The 2-closure of $X$ is the largest subgroup of $\operatorname{Sym}(\Omega)$ which preserves each orbital of $X$; denoted by $X^{(2)}$. We observe the following properties:

(i) $X$ is 2-transitive if and only if $X^{(2)}=\operatorname{Sym}(\Omega)$;

(ii) $X$ is primitive if and only if $X^{(2)}$ is primitive.

Assume that $X$ is imprimitive, and let $\mathcal{B}$ be an imprimitive partition of $\Omega$, that is, $\mathcal{B}$ is a non-trivial $X$-invariant partition of $\Omega$. For any block $B \in \mathcal{B}$, denote by $X^{B}$ the restriction of $X$ to $B$, that is, $X^{B}=\left\langle x \in X \mid B^{x}=B\right\rangle \leq \operatorname{Sym}(B)$. It is easily shown that an orbital of $X$ containing an $\operatorname{arc}(u, v)$ with $u, v \in B$ is also an orbital of $X^{(2)}$. It follows that $B$ is an imprimitive block for $X^{(2)}$, and $\mathcal{B}$ is an imprimitive partition of $\Omega$ for $X^{(2)}$. Thus we have the third observation:

(iii) $X$ and $X^{(2)}$ have the same imprimitive partitions of $\Omega$.

For the action induced on a block, we further have the following conclusion.

Lemma 2.1 ([18, 5.25]) Let $X$ be a transitive permutation group on $\Omega$ such that $\Omega$ has a non-trivial $X$-invariant partition $\mathcal{B}$. Then for $B \in \mathcal{B}$, we have

$$
\left(X^{(2)}\right)^{B} \leq\left(X^{B}\right)^{(2)}
$$

For some $c$-groups, the 2-closures are known.

Example 2.2 If $X$ is a primitive $c$-group of degree $n$, then either $X^{(2)}=\mathrm{S}_{n}$, or $n=p$ is a prime and $X \leq X^{(2)} \cong \mathbb{Z}_{p}: \mathbb{Z}_{l}$, where $l$ is a proper divisor of $p-1$.

A powerful method for studying $c$-groups comes from Schur ring theory, which was initiated by I. Schur and developed by H. Wielandt, see [17, Chapter 4]. A complete description of Schur rings over a cyclic group is given in $[4,11,12]$. From the description, the following result regarding $c$-groups may be derived.

Theorem 2.3 ([4, 11, 12]) Let $X$ be a c-group on $\Omega$, and let $X^{(2)}$ be the 2-closure of $X$. Then one of the following statements holds:

(i) $X^{(2)}=X_{1} \times X_{2} \times \cdots \times X_{r}$, where $r \geq 1$, each $X_{i} \cong \mathrm{S}_{n_{i}}$, or a normal c-group of degree $n_{i}$, such that $\left(n_{i}, n_{j}\right)=1$ and $n=n_{1} n_{2} \ldots n_{r}$;

(ii) $X$ has a normal subgroup $M$ such that each connected generalised orbital graph contains a subgraph $\Gamma$ which is an orbital graph of $X$ and is of the form $\Gamma=\Gamma_{M}\left[\overline{\mathrm{K}}_{b}\right]$, where $b=|M \cap G|$. 
The author is grateful to a referee for pointing out and formulating this theorem.

The next lemma shows that if $X$ contains an abelian regular subgroup, then each $X$ invariant partition of $\Omega$ consists of orbits of a normal subgroup.

Lemma 2.4 ([13, Lemma 3.1]) Let $X$ be a transitive permutation group on $\Omega$ which contains an abelian regular subgroup $G$. Let $\mathcal{B}$ be an $X$-invariant partition of $\Omega$ and $B \in \mathcal{B}$, and let $K$ be the kernel of $X$ acting on $\mathcal{B}$. Then $\mathcal{B}$ equals the set of $K$-orbits in $\Omega$, and $G \cap K$ is regular on $B$.

Now we are ready to prove Theorem 1.2. In the original version of this paper, a permutation group theoretical proof of Theorem 1.2 was given, which was independent of Theorem 2.3. The following proof was suggested by one of the referees. The author is grateful to him for this suggestion.

Proof of Theorem 1.2: Let $X$ be a $c$-group on $\Omega$ of degree $n$, and let $G$ be a regular cyclic subgroup of $X$. To complete the proof of Theorem 1.2, by Theorem 2.3, we may assume that the 2-closure $X^{(2)}$ satisfies

$$
X^{(2)}=X_{1} \times X_{2} \times \cdots \times X_{r},
$$

where $r \geq 1$, either $X_{i} \cong \mathrm{S}_{n_{i}}$, or $X_{i}$ is a normal $c$-group of degree $n_{i}$, such that $\left(n_{i}, n_{j}\right)=1$ and $n=n_{1} n_{2} \cdots n_{r}$.

If $r=1$ and $X_{1} \cong \mathrm{S}_{n_{1}}$, then $X^{(2)}=X_{1} \cong \mathrm{S}_{n_{1}}$, and hence $X$ is a 2-transitive $c$-group. If all $X_{i}$ 's are normal $c$-groups, then $X^{(2)}$ is a normal $c$-group. Since now $G \leq X \leq X^{(2)}$, it follows that $X$ is a normal $c$-group.

Thus we assume that $r \geq 2$, and that at least one of $X_{i}$ is a symmetric group, say $X_{1} \cong \mathrm{S}_{n_{1}}$, where $n_{1} \geq 4$. Let $Y_{i}=X \cap X_{i}$, and let $Y=\left\langle Y_{1}, Y_{2}, \ldots, Y_{r}\right\rangle$. Then $Y_{i} \triangleleft X$, and for $j \neq i$, we have $Y_{i} \cap Y_{j}=1$. Thus $Y=Y_{1} \times Y_{2} \times \cdots \times Y_{r}$, and $Y$ is normal in $X$. Let $G_{i}=G \cap X_{i}$. Then $\left|G_{i}\right|=n_{i}$, and for $j \neq i$, we have $G_{i} \cap G_{j}=1$. Since $\left(n_{i}, n_{j}\right)=1$, it follows that $G=G_{1} \times G_{2} \times \cdots \times G_{r}$.

Let $B$ be an orbit of $X_{i}$ in $\Omega$, and let $\mathcal{B}$ be the set of $X_{i}$-orbits in $\Omega$. Then $\mathcal{B}$ is an $X^{(2)}{ }_{-}$ invariant partition of $\Omega$. It is easily shown that $X_{i}$ equals the kernel of $X^{(2)}$ acting on $\mathcal{B}$. It follows that $\left(X^{(2)}\right)^{B} \cong X_{i}$. Now $G_{i}$ is cyclic and regular on $B$, and hence $X_{i}$ is a $c$-group of degree $n_{i}$.

Let $Y_{i}=X \cap X_{i}$. Since $G \leq X$, we have that $G_{i} \leq X \cap X_{i}=Y_{i}$. Hence $Y_{i}$ is a $c$-group on $B$, and $\mathcal{B}$ is the set of $Y_{i}$-orbits in $\Omega$. It is easily shown that $X^{B} \cong Y_{i}$. If $X_{i}$ is a normal $c$-group, then it follows that $Y_{i}$ is also a normal $c$-group.

Assume that $X_{i} \cong \mathrm{S}_{n_{i}}$. Then by Lemma 2.1, we have $\mathrm{S}_{n_{i}} \cong X_{i} \cong\left(X^{(2)}\right)^{B} \leq\left(X^{B}\right)^{(2)}$, and thus $X^{B}$ is 2-transitive. So $Y_{i} \cong X^{B}$ is a 2-transitive $c$-group of degree $n_{i}$.

Therefore, each $Y_{i}$ is a 2-transitive $c$-group or a normal $c$-group, of degree $n_{i}$, and $G \leq Y=Y_{1} \times Y_{2} \times \cdots \times Y_{r}$, completing the proof of Theorem 1.2.

\section{Acknowledgment}

This work forms a part of an Australian Research Council large grant project, and the author is supported by a QEII Fellowship. The author is very grateful to one referee for his 
constructive suggestions, and especially for his formulating Theorem 2.3 , which leads to the very short proof for Theorem 1.2 presented in this paper.

\section{References}

1. B. Alspach, M. Conder, D. Marušič, and M.Y. Xu, “A classification of 2-arc-transitive circulants,” J. Algebraic Combin. 5 (1996), 83-86.

2. C.Y. Chao, "On the classification of symmetric graphs with a prime number of vertices," Trans. Amer. Math. Soc. 158 (1971), 247-256.

3. C.Y. Chao and J.G. Wells, "A class of vertex-transitive digraphs," J. Combin. Theory Ser. B 14 (1973), $246-255$.

4. S.A. Evdokimov and I.N. Ponomarenko, "Characterization of cyclotomic schemes and normal Schur rings over a cyclic group, (Russian)," Algebra i Analiz 14(2) (2002), 11-55.

5. W. Feit, "Some consequences of the classification of finite simple groups," The Santa Cruz Conference on Finite Groups Univ. California, Santa Cruz, Calif., 1979, pp. 175-181, Proc. Sympos. Pure Math., 37, Amer. Math. Soc., Providence, R.I., 1980.

6. C.D. Godsil, "On the full automorphism group of a graph,” Combinatorica 1 (1981), 243-256.

7. D. Gorenstein, Finite Simple Groups, Plenum Press, New York, 1982.

8. G. Jones, "Cyclic regular subgroups of primitive permutation groups," J. Group Theory 5(4) (2002), $403-407$.

9. W. Kantor, Some consequences of the classification of finite simple groups, in Finite Groups-Coming of Age, John McKay (Ed.), Amer. Math. Soc., 1982.

10. I. Kovács, "Classifying arc-transitive circulants,” J. Algebraic Combin. 20 (2004), 353-358.

11. K.H. Leung and S.H. Man, "On Schur rings over cyclic groups," II, J. Algebra 183 (1996), 173-285.

12. K.H. Leung and S. H. Man, "On Schur rings over cyclic groups," Israel J. Math. 106 (1998), 251-267.

13. C.H. Li, "The finite primitive permutation groups containing an abelian regular subgroup," Proc. London Math. Soc. 87 (2003), 725-748.

14. C.H. Li, "Edge-transitive Cayley graphs and rotary Cayley maps," Trans. Amer. Math. Soc. (to appear).

15. C.H. Li, D. Marušič, and J. Morris, "A classification of circulant arc-transitive graphs of square-free order," J. Algebraic Combin. 14 (2001), 145-151.

16. J.X. Meng and J.Z. Wang, "A classification of 2-arc-transitive circulant digraphs," Disc. Math. 222 (2000), 281-284.

17. H. Wielandt, Finite Permutation Groups, Academic Press, New York, 1964.

18. H. Wielandt, Permutation groups through invariant relations and invariant functions, in Mathematische Werke/Mathematical works. Vol. 1. Group theory, Bertram Huppert and Hans Schneider (Eds.), Walter de Gruyter Co., Berlin, 1994, pp. 237-266.

19. M.Y. Xu, H.S. Sim, and Y.J. Baik, "Arc-transitive circulant digraphs of odd prime-power order," Discrete Math. 287 (2004), 113-119. 